\title{
ROLE OF OLIGODENDROCYTES IN REVERSING ETHAMBUTOL INDUCED OPTIC NEUROPATHY
}

Owembabazi Elna* ${ }_{1,2}$, Ahumuza Ronald, Juma Ochieng John1, Buzinde Isaac1, Ssengendo Ibrahim1, Ssempijja Fred 1.

1Department of Anatomy, Kampala International University Western Campus, P.O.Box 71 IshakaBushenyi, Uganda.

${ }_{2}$ School of Anatomical Science, University of the Witwatersrand, 29 Princess of Wales Terrace, Johannesburg, South Africa

Correspondence to Elna Owembabazi Department of Anatomy, Kampala International University Western Campus, P.O.Box 71 Ishaka-Bushenyi, Uganda. Phone: +27626960405. Email: lenaoet@gmail.com

\section{ABSTRACT}

Ethambutol is one of the first line drugs for treating tuberculosis. Its toxic effects on the optic nerve are majorly classified as reversible. However, worsening of vision and permanent vision loss after ethambutol discontinuation is also documented. To determine the effect of ethambutol toxicity on oligodendrocytes at different periods of treatment. Twenty-five male adult wistar rats of $110-130 \mathrm{~g}$ average weight were housed in cages, exposed to 12-hour of dark and light cycles. After one week of acclimatization, five animals were randomly selected and sacrificed prior to ethambutol treatment for the control group (week 0). The remaining 20 animals were each orally administered $100 \mathrm{mg} / \mathrm{kg} /$ day ethambutol. Five animals were randomly picked and sacrificed at the end of first, second, third, and fourth week of ethambutol treatment. There was no statistically significant difference in the number of oligodendrocyte cells obtained at the different stages of ethambutol treatment. Oligodendrocytes are not vulnerable to ethambutol toxicity for at least one month and they play a key role in reversing ethambutol induced neuropathy through myelin sheaths reconstruction.

Key Words: Ethambutol, Oligodendrocytes, Optic Nerve, Optic Neuropathy.

\section{INTRODUCTION}

Ethambutol (EMB) also known as Myambutol, is one of the drugs for treating tuberculosis (TB) (Somasundaram et al., 2014). Worldwide, one-third of the population is affected by tuberculosis (The Lancet Infectious Diseases, 2011). The development of multidrug resistant TB along with Human Immunodeficiency Virus/Acquired Immunodeficiency Syndrome condition have greatly increased the incidence of TB, making it a primary public health threat (Gray and Cohn, 2013). Ethambutol is the only effective first line anti-tuberculosis agent that prevents development of resistance to companion drugs and broadening of the resistance spectrum (Orikiriza et al., 2015).

Soon after its discovery in 1960s (Forbes et al., 1962), EMB was reported to cause changes in the optic nerve structure that result into optic neuropathy. Damage to the optic nerve structure is one of the most serious conditions because of the inability of the fibres to regenerate (Tawse et al., 2014), posing a risk of permanent and potentially severe loss of vision (Boulanger et al., 2013). Optic nerve consists of the myelinated axons of the retinal ganglion cells (de Lima et al., 2012), these mediate for the special sense of vision via the Special somatic afferent fibre (Watson, 2014). Oligodendrocytes, the major neuroglia in the optic nerve are responsible for production of myelin sheaths of the nerve fibres (Simons and Nave, 2016), which facilitates the rapid conduction of axons(Chamberlain et al., 2016)

Ethambutol induced toxic optic neuropathy is a symmetrical progressive painless condition, presenting with symptoms such as; blurring of vision, decrease in visual acuity (Rasool et al., 2015), bitemporal visual field scotoma defects, centro-caecal scotoma and disturbances in colour perception (Han et al., 2015). Different experimental animal studies using toxic doses of EMB have reported vacuolations, axonal fragmentation, inflammatory changes, demyelination and central necrosis in the optic nerve (Osaguona et al., 2014; Yang et al., 2016). 
Although EMB toxicity is classically described as reversible on discontinuation with complete recovery over period of weeks to months (Holla et al., 2015), worsening of vision after EMB discontinuation has been observed in some patients (Garg et al., 2015). Previous studies have reported severe, and irreversible vision loss despite frequent monitoring and standard dosages (Chen, Lin, and Sheu, 2015).
Moreover, apart from stopping the use of EMB, there is no specific treatment available for alleviating EMB induced toxic optic neuropathy (Rasool et al., 2015). This study aimed at exploring the role of oligodendrocytes in reversing this condition by determining the effect of ethambutol toxicity on oligodendrocytes at different periods of treatment.

\section{MATERIALS AND METHODS}

Ethical clearance for this study was given by the Directorate of Postgraduate Studies and Research of Kampala International University, Uganda. All experimental procedures on the animal were conducted in accordance with the 2010 National Research Council Guide for Care and use of Laboratory Animals.

A total of 25 male adult wistar rats of $110-130 \mathrm{~g}$ average weight were used in this study. The animals were housed in cages, exposed to 12hour of dark and light cycles. They were fed on pellets and provided with tap water ad libitum. Treatment with ethambutol started after one week of acclimatization. Administration of ethambutol was performed as previously described (Elna et al., 2018), and doses of $100 \mathrm{mg} / \mathrm{kg} /$ day were used to induce visual impairment in the experimental animals. Five animals were randomly selected and sacrificed prior to ethambutol treatment for the control group (week 0 ). Five animals were randomly picked and sacrificed at the end of 1, 2, 3, 4 weeks of ethambutol treatment.

Obtaining the optic nerves and tissue processing were performed as previously described (Owembabazi et al., 2017) Sections of $5 \mu \mathrm{m}$ thickness were cut using an ultra- microtome, and mounted on salinized glass slides. These were heat fixed, put in xylene for 3 minutes to remove paraffin wax and then put in two containers of absolute alcohol for 30 seconds each to remove the xylene. They were then transferred to $90 \%$ alcohol for 30 seconds and to $70 \%$ alcohol for 30 seconds and rinsed in distilled water. The clean slides were then put in toluidine blue stain for 2 minutes, after which they were washed thoroughly in tap water. They were then dehydrated through $95 \%$ and $100 \%$ alcohols, then cleared in xylene and a coverslip was immediately put (Ephros, 2004). The slides were examined under a light microscope, the number of oligodendrocytes were counted per unit area of $0.50 \mathrm{~mm} 2$, after calibrating the ocular micrometre with stage linear micrometre, using X40 objective.

Using graph pad prisms $V 6$, mean \pm SEM were obtained and analysis of variance (ANOVA) was applied to determine group mean differences in the number of oligodendrocytes between the control and test groups. A post-HOC Tuckey test was used to determine which means differed, considering a p-value of $<0.05$ to be statistically significant.

\section{RESULTS}

Stained cross sections of the optic nerves were observed under light microscope, the oligodendrocytes were identified as small cells with a central dark nucleus and a rim of unstained cytoplasm. The average number of oligodendrocytes cells at the different stages of ethambutol treatment were; week 1:
22.4 \pm 2.4 , week 2: $22.4 \pm 2.34$, week 3 : $23.4 \pm 1.03$, and week $4: 21 \pm 1.98$. When these were each compared to $23 \pm 1.41$ that was obtained in the animals that were not treated with ethambutol (week 0 ), the differences were all statistically insignificant $(p>0.05)$. 


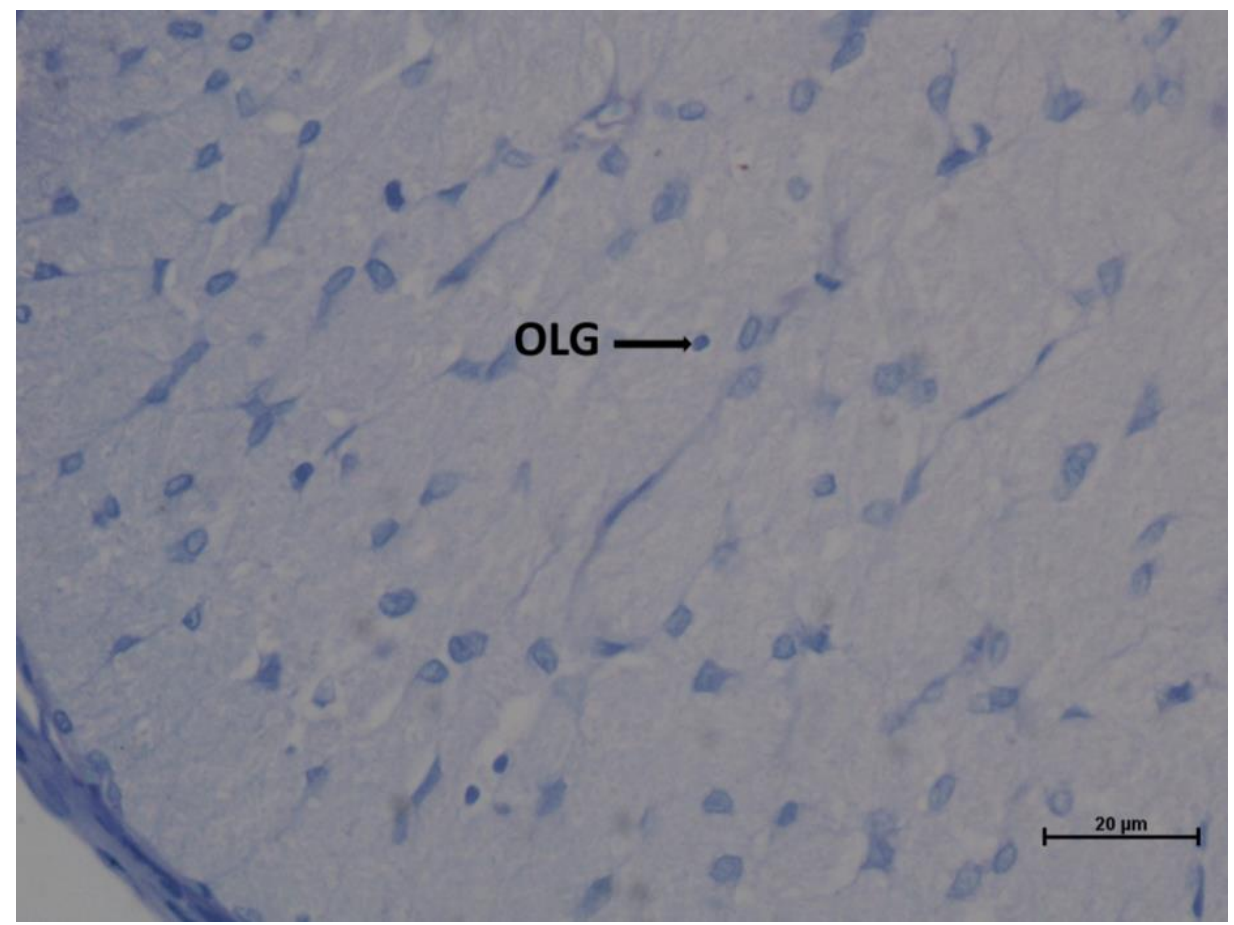

Figure 1: Photomicrographs of Optic Nerve Cross Section, arrow pointing at oligodendrocyte cell (OLG). Toluidine blue stain Stain, X40.

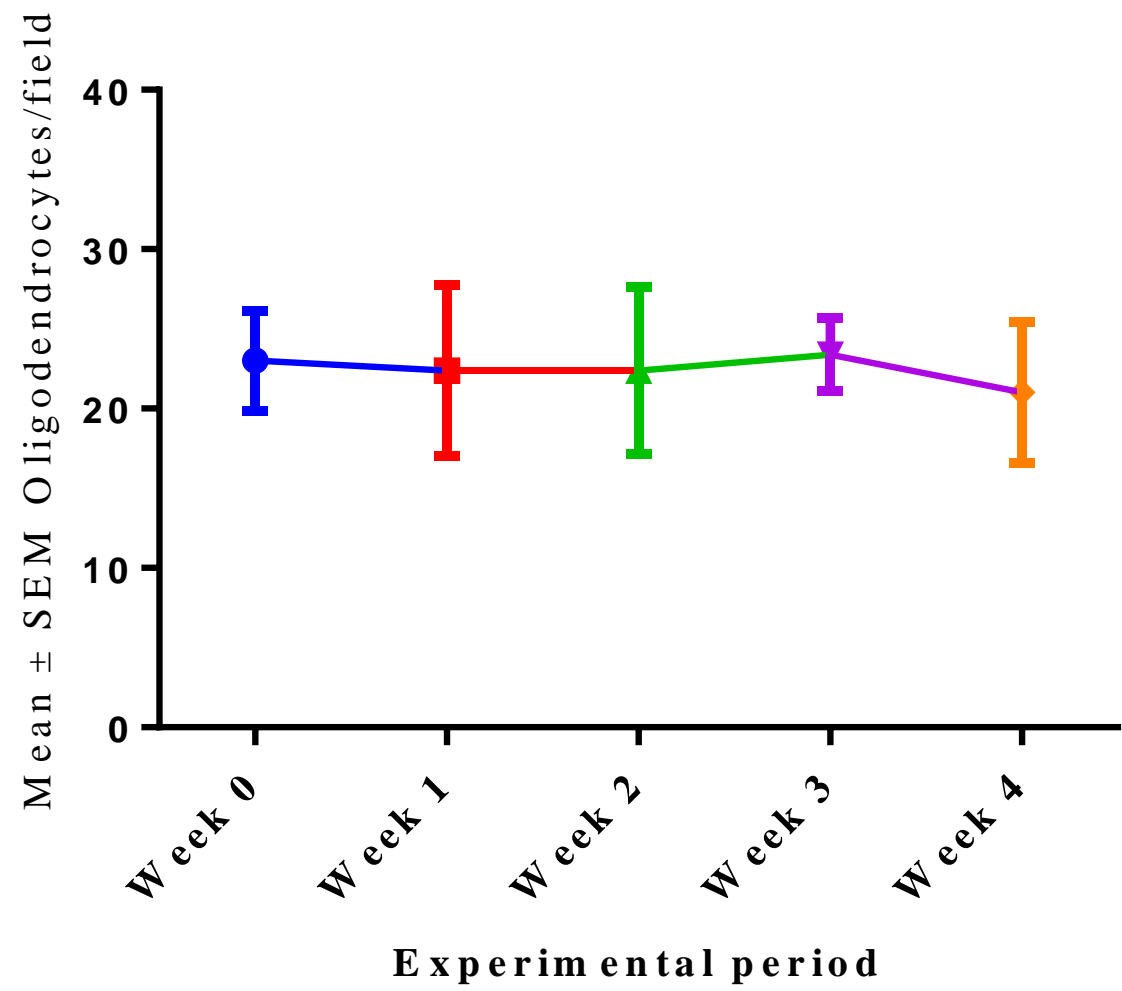

Figure 2: A line graph showing the number of oligodendrocyte cells at different periods o the experiment. The $p$ values comparison against the negative control (Week 0); Week $1=0.9980$, Week $2=0.9980$, Week $3=0.9997$, Week $4=0.8701$. 


\section{DISCUSSION}

In this study, we investigated the effect of ethambutol toxicity on oligodendrocytes to explore their role in reversing ethambutol induced toxic optic neuropathy. Our results (figure 1) showed no significant differences in all preparations after different periods of treatment. This is consistent with earlier findings; reporting no significant changes in the number of oligodendrocytes after a month of ethambutol treatment in experimental animals (Kinoshita et al., 2012). However, a recent study reported optic nerve oligodendropenia in rabbits after 6 weeks of administering $100 \mathrm{mg} / \mathrm{kg} /$ day of ethambutol (Zada and Badar, 2018).

Oligodendrocytes deposit myelin around the axons (Simons and Nave, 2016). This enables fast transmission of signals through the nerves in central nervous system (Chamberlain et al., 2016). Ethambutol toxicity damages myelin, causing progressive axonal loss that results into ethambutol induced optic neuropathy (Talbert and Sadun, 2010). Visual impairment has also been reported in some neurological disorders such as; multiple sclerosis that cause extensive demyelination (Balcer et al., 2015). Based on our finding, oligodendrocytes are not vulnerable to ethambutol toxicity for at least one month. Therefore, they are able to reconstruct the myelin sheaths around the optic nerve axons after ethambutol discontinuation and thus take part in reversing ethambutol induced neuropathy.

In conclusion, the number and integrity of oligodendrocytes are both vital in the process of reversing ethambutol induced optic neuropathology. We stipulate that patients who develop severe, irreversible vision loss despite EMB discontinuation, most likely had existing oligodendrocyte cell disorders.

We recommend that patients with demyelinating diseases that are undergoing ethambutol treatment are put under visual impairment monitoring. Further studies should determine the neuroglia genetic variations in the patients that develop irreversible ethambutol induced optic neuropathy.

Competing interest: Authors declare that there exist no competing interests.

\section{REFERENCES}

1. Balcer L. 2015. Vision and vision-related outcome measures in multiple sclerosis. Brain. doi: 10.1093/brain/awu335.

2. Boulanger Scemama E, Touitou V, Le Hoang P. 2013. '[Bitemporal hemianopia as presenting sign of severe ethambutol toxicity]. J Fr Ophtalmol, 36, e163-7. doi: 10.1016/j.jfo.2012.12.008.

3. Chamberlain KA. 2016. Oligodendrocyte regeneration: Its significance in myelin replacement and neuroprotection in multiple sclerosis. Neuropharmacology. doi: 10.1016/j.neuropharm.2015.10.010.

4. Chen SC, Lin MC, Sheu SJ. 2015. Incidence and prognostic factor of ethambutol-related optic neuropathy: 10-year experience in southern Taiwan', Kaohsiung Journal of Medical Sciences, 31: 358-362. doi: 10.1016/j.kjms.2015.05.004.

5. Elna O. 2018. Lantana Trifolium Ethanolic Extract has a Protective Potential against Ethambutol Induced Changes in Visual Acuity. SMJ Clinical Anatomy. 2(3): 3-6.

6. Ephros HD. 2004. Toluidine blue staining. Journal of Oral and Maxillofacial Surgery. doi: 10.1016/j.joms.2004.05.005.

7. Forbes M, Kuck NA, Peets EA. 1962. Mode of action of ethambutol. Journal of bacteriology. 84:1099-1103.

8. Garg P. 2015. A prospective study of ocular toxicity in patients receiving ethambutol as a part of directly observed treatment strategy therapy.', Lung India : official organ of Indian Chest Society, 32: 16-19. doi: 10.4103/0970-2113.148428.

9. Gray JM, Cohn DL. 2013. Tuberculosis and HIV coinfection', Seminars in Respiratory and Critical Care Medicine. doi: 10.1055/s-0032-1333469.

10. Han J. 2015. Longitudinal analysis of retinal nerve fiber layer and ganglion cell-inner plexiform layer thickness in ethambutol-induced optic neuropathy.', Graefe's archive for clinical and 
experimental ophthalmology $=$ Albrecht von Graefes Archiv fur klinische und experimentelle Ophthalmologie, 253(12): 2293-2299. doi: 10.1007/s00417-015-3150-8.

11. Holla SN. 2015. Cycloserine induced late onset psychosis and ethambutol induced peripheral neuropathy associated with MDR-TB treatment in an Indian patient- A rare case report', Journal of Clinical and Diagnostic Research, 9: FD01-FD03. doi: 10.7860/JCDR/2015/12417.5588.

12. Kinoshita J. 2012. Retinal function and morphology in monkeys with ethambutol-induced optic neuropathy', Investigative Ophthalmology and Visual Science, 53: 7052-7062. doi: $10.1167 /$ iovs.12-10308.

13. de Lima S. 2012. Full-length axon regeneration in the adult mouse optic nerve and partial recovery of simple visual behaviors', Proceedings of the National Academy of Sciences. doi: 10.1073/pnas.1119449109.

14. Orikiriza P. 2015. Low resistance to first- and second-line anti-tuberculosis drugs among treatment naive pulmonary tuberculosis patients in Southwestern Uganda. PLOS ONE. doi: 10.1371/journal.pone.0118191.

15. Osaguona VB. 2014. Optic Chiasm Involvement on MRI With Ethambutol-Induced Bitemporal Hemianopia', Journal of Neuro-Ophthalmology, 34: 155-158. doi: 10.1097/WNO.0000000000000095.

16. Owembabazi E. 2017. Neuroprotective Potential of Lantana Trifolium Ethanolic Extract against Ethambutol Induced Histological Changes in the Optic Nerve', Anatomy Journal of Africa, 6: 1071-1079.

17. Rasool M. 2015. Determination of potential role of antioxidative status and circulating biochemical markers in the pathogenesis of ethambutol induced toxic optic neuropathy among diabetic and non-diabetic patients. Saudi Journal of Biological Sciences. 22: 739-743. doi: 10.1016/j.sjbs.2014.09.019.

18. Simons M, Nave KA. 2016. Oligodendrocytes: Myelination and axonal support', Cold Spring Harbor Perspectives in Biology. doi: 10.1101/cshperspect. a020479.

19. Somasundaram S, Ram A, Sankaranarayanan L. 2014. Isoniazid and Rifampicin as Therapeutic Regimen in the Current Era: A Review. Journal of Tuberculosis Research. doi: 10.4236/jtr.2014.21005.

20. Talbert Estlin KA, Sadun AA. 2010. Risk factors for ethambutol optic toxicity', International Ophthalmology, 30: 63-72. doi: 10.1007/s10792-009-9293-z.

21. Tawse K L. 2014. Optical coherence tomography shows retinal abnormalities associated with optic nerve disease', Br J Ophthalmol, 98 Suppl 2: ii30-3. doi: 10.1136/bjophthalmol-2013304301.

22. The Lancet Infectious Diseases. 2011. 'The worldwide epidemic of multidrug-resistant tuberculosis'. doi: 10.1016/S1473-3099(11)70105-0.

23. Watson AB. 2014. A formula for human retinal ganglion cell receptive field density as a function of visual field location. Journal of Vision. doi: 10.1167/14.7.15.

24. Yang HK. 2016. Incidence of toxic optic neuropathy with low-dose ethambutol', The International Journal of Tuberculosis and Lung Disease. doi: 10.5588/ijtld.15.0275.

25. Zada N, Badar A. 2018. Histological Grading of Ethambutol Induced Optic Toxicity in Rabbits', 8: 21-25. 